\title{
Classification of robust cycle bases and relations to fundamental cycle bases
}

\author{
Alexander Reich \\ Mathematics Institute, Brandenburg University of Technology, \\ Platz der Deutschen Einheit 1, D-03046 Cottbus, Germany
}

Received 29 September 2016, accepted 15 September 2017, published online 10 November 2017

\begin{abstract}
The construction of a cycle in a graph can be realized by iteratively adding cycles of a cycle basis. The construction of each elementary cycle is only possible if this cycle basis is robust. In the last years, different classes of robust cycle bases have been established. We compare these classes and show that they are completely unrelated. More precisely, we draw a Venn diagram which displays the obvious containedness relations and show that each of its regions is not empty. In addition, we continue the comparison with fundamental cycle bases.
\end{abstract}

Keywords: Minimum cycle basis, robust cycle basis, quasi-robust cycle basis, fundamental cycle basis.

Math. Subj. Class.: 05C10, 05C38, 05C50

\section{Introduction}

Cycle bases of graphs have numerous applications, e.g. in the fields of periodic timetable optimization [9], coordination of traffic signals [15], or chemistry [4]. The first reference [9] additionally provides a useful classification of several types of cycle bases utilized for computations in the mentioned areas. The author considered the seven classes of directed, undirected, integral, totally unimodular, planar, as well as weakly and strictly fundamental cycle bases and compared them to each other.

Another line of research has been initiated by Kainen [6] who investigated robust cycle bases. Strengthening and weakening the concept of robust cycle bases led to four different types of robust cycle bases, which were further studied in [8] and [12], and recently in [7]. The latter paper provides an application of robust cycle bases to the analysis of commutative diagrams in groupoids.

E-mail address: alexanderreich@arcor.de (Alexander Reich) 
Similarly to the work of Liebchen [9], we show in our paper that no two of these four classes coincide and give a separating example for each pair of the classes. All of our examples provide a graph with its uniquely minimum cycle basis. This indicates that each class of robust cycle bases admits its own minimization problem.

A further focus in this paper is the relationship of robust and fundamental cycle bases, The investigation on this topic has been initiated in [8]. We continue this research by providing more examples of cycle bases which are even minimum in almost all cases. We are able to eliminate one of two question marks in a map given there, where the authors conjectured the existence of examples.

The results in this paper appeared also in the thesis [13].

\section{Preliminaries}

Throughout the paper, we consider only simple undirected weighted graphs $G=(V, E)$ with finite node set $V(G)=V$, finite edge set $E(G)=E$, and weight function $w: E \rightarrow$ $\mathbb{R}_{>0}$. The degree of a node $v \in V$ is denoted by $\operatorname{deg}(v)$. A path $P$ of length $\ell$ in a graph is a sequence $P=\left(v_{0}, v_{1}, \ldots, v_{\ell}\right)$ of pairwise disjoint nodes with $v_{i-1} v_{i} \in E$ for $1 \leq i \leq \ell$. The length of a shortest path between two nodes $u$ and $v$ in $G$ is called the distance $\operatorname{dist}_{G}(u, v)$. A path from node $u$ to node $v$ is referred to as $u$-v-path.

A circuit $C$ in $G$ is a non-empty connected subgraph of $G$ with $\operatorname{deg}(v)=2$ for all $v \in V(C)$. We define $|C|:=\sum_{e \in E(C)} w(e)$ as the length of a circuit $C$. A cycle $Z$ in $G$ is a subgraph of $G$ where $\operatorname{deg}(v)$ is even for all $v \in V(Z)$.

For a spanning tree $T=(V, E(T))$ of $G$ and an edge $e \in E \backslash E(T)$ define the fundamental circuit $C_{T}(e)$ as the unique circuit in $(V, E(T) \cup\{e\})$. The non-tree edges are also called chords of the spanning tree $T$. We usually identify circuits, cycles, and trees with their edge sets.

The cycle space $\mathcal{C}(G)$ of a graph $G=(V, E)$ is the vector subspace of $\mathrm{GF}(2)^{E}$ that is generated by the incidence vectors of the circuits in $G$. The sum of two cycles $Z_{1}$ and $Z_{2}$ in this vector space is their symmetric difference $\left(Z_{1} \cup Z_{2}\right) \backslash\left(Z_{1} \cap Z_{2}\right)$. A cycle basis $B$ of $G$ is a set of $\nu=m-n+1$ circuits whose incidence vectors form a basis of $\mathcal{C}(G)$. The size $\Phi(B)$ of a cycle basis $B$ is defined as $\Phi(B):=\sum_{C \in B}|C|$. A cycle basis $B$ of $G=(V, E)$ is designated strictly fundamental iff there is a spanning tree $T=(V, E(T))$ with $B=\left\{C_{T}(e) \mid e \in E \backslash E(T)\right\}$.

A cycle basis $B=\left\{Z_{1}, \ldots, Z_{\nu}\right\}$ is weakly fundamental if there exists a permutation $\pi \in S_{\nu}$ such that

$$
Z_{\pi(i)} \backslash \bigcup_{j=1}^{i-1} Z_{\pi(j)} \neq \emptyset \quad \text { for all } i=2, \ldots, \nu .
$$

Weakly fundamental cycle were also the matter of [5] where the authors characterized graphs for which every cycle basis is weakly fundamental.

If $B$ is a cycle basis then every cycle $Z$ has a unique representation $Z=\sum_{C \in B} \lambda_{C} C$ with $\lambda_{C} \in\{0,1\}$. The subset $\left\{C \in B \mid \lambda_{C}=1\right.$ and $\left.Z=\sum_{C \in B} \lambda_{C} C\right\}$ is called the support $\operatorname{supp}(Z)$.

The following simple lemma is needed to justify the minimality of some of our cycle bases.

Lemma 2.1. For a given strictly fundamental cycle basis $B$ of an undirected graph $G=$ $(V, E)$ one can always find a weight function $w$ such that $B$ is the unique minimum cycle 
basis of $G$.

Proof. Let $T$ be a fundamental spanning tree which induces $B$. For every edge $e \in T$ set $w(e)=1$. Define $d:=\max \left\{\operatorname{dist}_{T}(u, v) \mid u v \in E \backslash T\right\}$ and $\operatorname{assign} w(e)=2 d-\operatorname{dist}_{T}(u, v)$ for the remaining edges $e=u v$. Observe that the minimum of $w$ restricted to the chords is $d$. Now, every circuit in $B$ has a weight of $2 d$ while all other cycles of $G$ have a greater weight since they contain at least two chords and at least one tree edge or at least three chords.

For Example 5.5, the following enhancement of Lemma 2.1 is necessary.

Lemma 2.2. For a given strictly fundamental cycle basis $B$ of an undirected graph $G=$ $(V, E)$ one can always find a weight function $w$ such that $B$ is the unique minimum cycle basis of $G$ and such that there is a chord $e=u v$ with $w(e)<\operatorname{dist}_{T}(u, v)$.

Proof. The proof has essentially the same structure as the proof of Lemma 2.1. Thus, set $w(e)=1$ for all tree edges of a given fundamental spanning tree $T$ which induces $B$. And again, let $d:=\max \left\{\operatorname{dist}_{T}(u, v) \mid u v \in E \backslash T\right\}$. For the edges $e=u v$ in $E \backslash T$, we now assign the weight $w(e)=2 d-\operatorname{dist}_{T}(u, v)-\varepsilon$, for an $\varepsilon>0$ whose value is determined later. The minimum of $w$ restricted to the chords is $d-\varepsilon$, and each circuit $C \in B$ has the weight $w(C)=2 d-\varepsilon$.

Now, look at a circuit which is not in $B$. It consists of $c \geq 2$ chords and $t \geq 0$ tree edges. Furthermore, $c=2$ implies $t \geq 1$. The length of the circuit is at least $c(d-\varepsilon)+t$. For all $\varepsilon \in\left(0, \frac{(c-2) d+t}{c-1}\right)$, this value is greater than $2 d-\varepsilon$, i.e. greater than the weight of a basic circuit. Because $c \geq 2$, the denominator of the upper endpoint of the interval is not zero, and since $c+t \geq 3$, also the numerator is not zero. Hence, this interval is not empty and we can take any $\varepsilon$ from this interval.

Finally, for a chord $e=u v$ with $\operatorname{dist}_{T}(u, v)=d$, the weight $w(e)$ has the value $d-\varepsilon<\operatorname{dist}_{T}(u, v)$.

\section{Classes of robust cycle bases}

In order to define the four different types of robust cycle bases, we essentially follow the exposition in [12]. Similarly as there, we need at first the concept of (strictly) well-arranged sequences of circuits. Afterwards, we deduce several simple inclusions and present a map of the relationship between the different classes of robust cycle bases.

Definition 3.1 ((Strictly) well-arranged sequence). A sequence $S=\left(C_{1}, \ldots, C_{k}\right)$ of circuits in an undirected graph is called well-arranged if for all $j=1, \ldots, k$ the $\mathrm{GF}(2)$-sum $\sum_{i=1}^{j} C_{i}$ is also a circuit. A well-arranged sequence of circuits is strictly well-arranged if for all $j=2, \ldots, k$ the intersection $C_{j} \cap \sum_{i=1}^{j-1} C_{i}$ is a single path.

The path in Definition 3.1 contains at least one edge. Otherwise, the sum $C_{j}+\sum_{i=1}^{j-1} C_{i}$ was not a circuit and thus, the sequence was not even well-arranged, at all. It is clear that every strictly well-arranged sequence is also well-arranged. Furthermore, it is known that there are well-arranged sequences that are not strictly well-arranged. The authors of [8] provide such an example in which the sum of two basic circuits is again a circuit, but they intersect in three paths. Note that it is not forbidden that a circuit appears more than once in a (strictly) well-arranged sequence. 
With this in mind, we are now able to define the four different types of robust cycle bases which were developed in [12].

Definition 3.2 (Cyclically/strictly robust and (strictly) quasi-robust cycle basis). A cycle basis $B$ of a graph $G$ is (strictly) quasi-robust if for each circuit $C$ in $G$ there is a (strictly) well-arranged sequence $S_{C}=\left(C_{1}, \ldots, C_{k-1}, C_{k}\right)$ such that $C=\sum_{i=1}^{k} C_{i}$ and $C_{i} \in B$ for $i=1, \ldots, k$. A strictly quasi-robust cycle basis is strictly robust if the circuits in the strictly well-arranged sequence are pairwise disjoint. Analogously, a quasi-robust cycle basis is cyclically robust if the according well-arranged sequence does not contain a circuit twice. If we do not want to specify the particular type of robustness, we simply speak about a robust cycle basis.

It can be concluded that for strictly and for cyclically robust cycle bases the wellarranged sequence of a circuit $C$ must not contain basic circuits which are not in the support of $C$. Also, directly from these definitions, we can immediately derive the following facts:

- every strictly quasi-robust cycle basis is quasi-robust,

- every strictly robust cycle basis is strictly quasi-robust,

- every strictly robust cycle basis is cyclically robust, and

- every cyclically robust cycle basis is quasi-robust.

These inclusions hold since in each case, we require additional properties for the more specific class. The inclusions give rise to the diagram in Figure 1.

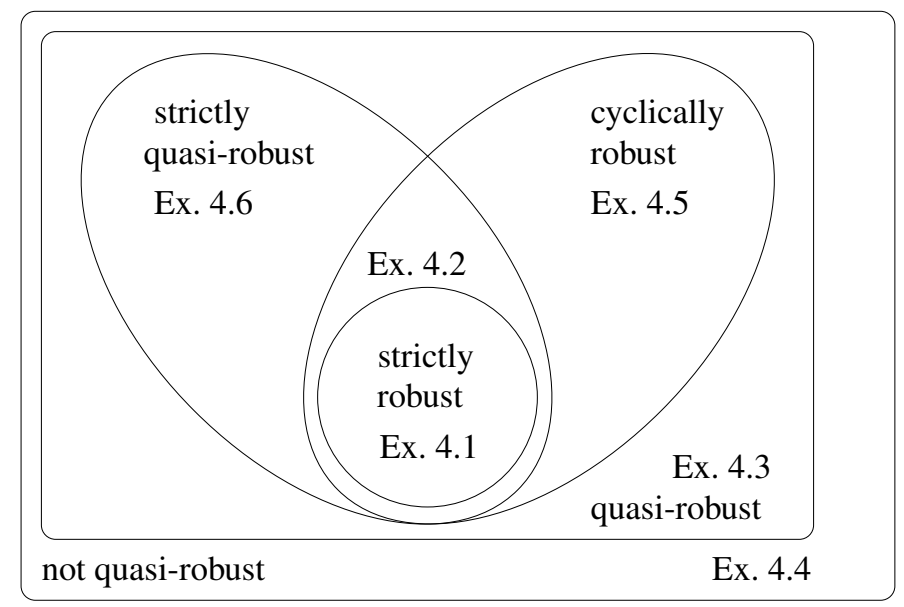

Figure 1: Map of robust cycle bases.

Not much is known about which graph classes can have which type of robust cycle bases. Furthermore, it is unknown whether each graph admits a robust cycle basis of any of the four types. Table 1 summarizes the related results. To the best of our knowledge, these are the only known ones. 
Table 1: Summary of known graph classes for which the stated type of cycle bases is guaranteed.

\begin{tabular}{llr}
\hline Graph class & Robustness & Reference \\
\hline planar graphs & strictly robust & {$[2]$} \\
complete graphs & strictly robust & {$[6]$} \\
complete bipartite graphs $K_{m, n}$ with $m \leq 4$ and $n \leq 5$ & strictly robust & {$[12]$} \\
general complete bipartite graphs & quasi-robust & {$[12]$} \\
wheels & cyclically robust & {$[8]$} \\
\hline
\end{tabular}

\section{Examples of robust cycle bases}

In this section, we show that the inclusions derived in the last section are valid only in the given direction. Thus, no two of the classes are equivalent. To point this out, we give an example of a graph and a cycle basis for each region in the map in Figure 1 and thus show that it is not empty.

Except in Example 4.2, all cycle bases are strictly fundamental. According to Lemma 2.1, we can choose a weight function such that this cycle basis is the unique minimum cycle basis on this graph. However, the given cycle basis in Example 4.2 is also the unique minimum one. The existence of a graph with a minimum cycle basis in each region of the map indicates that each class - actually even each non-empty difference of two classes - of robust cycle bases admits its own minimization problem.

Remember that we do not know an efficient algorithm for the computation or for the recognition of any type of robust cycle bases on general graphs. Thus, to prove a cycle basis of a graph $G$ as (strictly) quasi-robust, we have to indicate a (strictly) well-arranged sequence of basic circuits for every circuit in $G$. Analogous sequences have to be found for (strictly) robust cycle bases. In the latter case, a basic circuit is allowed to occur at most one time in each of these sequences.

On the other hand, a cycle basis $B$ of a graph $G$ is not quasi-robust if there exists a circuit $C^{\prime}$ in $G$ such that for each $C \in B$ the sum $C^{\prime}+C$ is not a circuit. To show that the cycle basis is not strictly quasi-robust, one has to verify that the cut $C^{\prime} \cap C$ does not form a path for one circuit $C^{\prime}$ in the graph and for all $C \in B$. Finally, to show that a cycle basis is not a cyclically robust or a strictly robust cycle basis it suffices to check only the circuits of the support of such a circuit $C^{\prime}$.

We now start with the description of the examples.

Example 4.1 (Strictly robust cycle basis). The first example is the simple graph $C_{3}$ that consists of exactly one circuit of length 3. Clearly, its unique cycle basis is strictly robustand strictly fundamental and minimum, as well.

Example 4.2 (Cyclically robust and strictly quasi-robust cycle basis-not strictly robust). Our second example is the complete bipartite graph $K_{3,3}$, see Figure 2 (a). The cycle basis $B=\left\{C_{1}, C_{2}, C_{3}, C_{4}\right\}$ is highlighted in Figure 2 (b). It is not strictly fundamental, thus, we suggest the indicated weights to make the cycle basis minimum. All other circuits have a greater weight. The weights of all circuits are denoted below the graph in Figures 2 (b), (c) and (d). We show that $B$ is cyclically robust and strictly quasi-robust, but not strictly robust. For $2 \leq k \leq \nu$ we denote $C_{i_{1}, \ldots, i_{k}}:=\sum_{j=1}^{k} C_{i_{j}}$. 


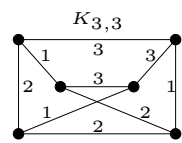

(a)

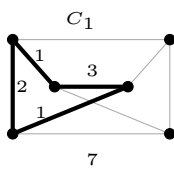

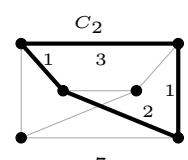

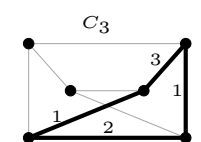

7

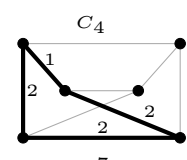

(b)

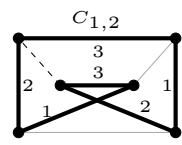

12

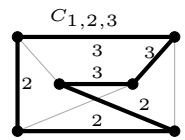

15

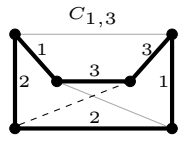

12

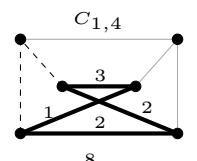

(c)

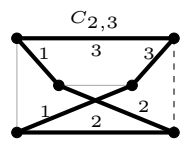

12

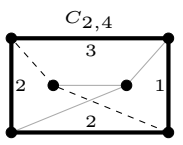

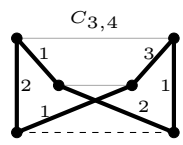

10

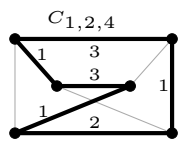

11

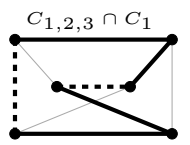

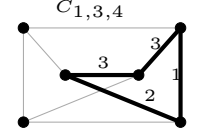

9

(d)

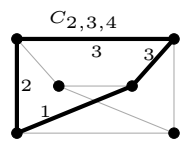

9

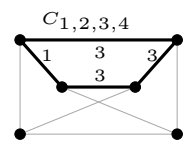

10
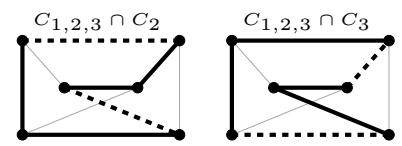

(e)

Figure 2: The $K_{3,3}$ with weights on the edges (a). The four basic circuits and their weights below (b). All other circuits and their weights (c) and (d). The intersections (dashed edges) of $C_{1,2,3}$ with the circuits of its support (e).

Cyclically robust. The $K_{3,3}$ is cubic, hence it contains no cycle with vertices of degree 4 or more. Furthermore, it has only six vertices, but it is triangle free. Thus, there is no cycle consisting of two triangles. This means that every cycle is a circuit and therefore, each cycle basis of $K_{3,3}$ is cyclically robust.

Not strictly robust. The given basis is not strictly robust, since there is no strictly wellarranged sequence for $C_{1,2,3}$, in which every basic circuit occurs only once. Observe this by looking at Figure 2 (e). It is indicated that $C_{1,2,3}$ has an intersection consisting of two path (dashed edges) with each circuit from its support.

Strictly quasi-robust. For the circuits which have exactly two basic circuits in their supports, these two circuits intersect in a single path, illustrated by the dashed edges in Figure 2 (c). For the circuits depicted in Figure 2 (d) we provide the sequences $S_{C_{1,2,3}}=$ $\left(C_{1}, C_{3}, C_{4}, C_{2}, C_{4}\right), S_{C_{1,2,4}}=\left(C_{1}, C_{4}, C_{2}\right), S_{C_{1,3,4}}=\left(C_{1}, C_{3}, C_{4}\right), S_{C_{2,3,4}}=\left(C_{2}, C_{4}\right.$, $\left.C_{3}\right)$, and $S_{C_{1,2,3}, 4}=\left(C_{1}, C_{3}, C_{4}, C_{2}\right)$, which are all strictly well-arranged. Hence, the cycle basis is strictly quasi-robust.

Example 4.3 (Quasi-robust cycle basis-neither cyclically robust nor strictly quasi-robust). This example is borrowed from [6]. We consider the complete bipartite graph $K_{5,5}$, the strictly fundamental cycle basis $B$ induced by the spanning tree $T$ shown in Figure 3 (a), and the circuit $C$ aside in Figure 3 (b). The sixteen basic circuits themselves are also de- 
picted in Figure 4 as black edges. Assigning weights according to Lemma 2.1, $B$ becomes the unique minimum cycle basis.

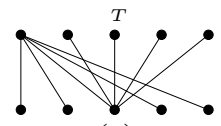

(a)

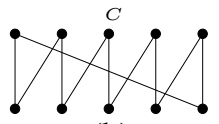

(b)

Figure 3: Spanning tree $T$ of $K_{5,5}$ (a). The circuit $C$ considered in the text (b).
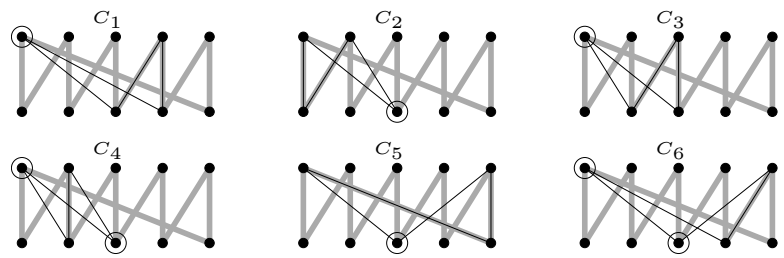

(a)
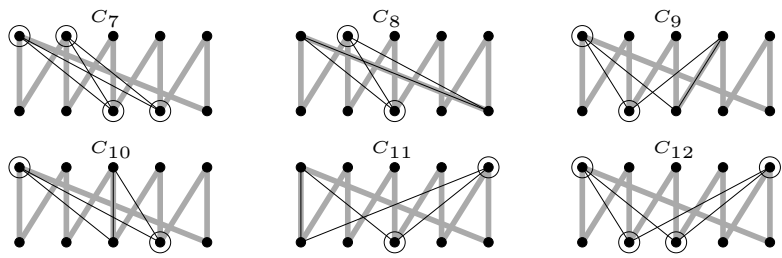

(b)
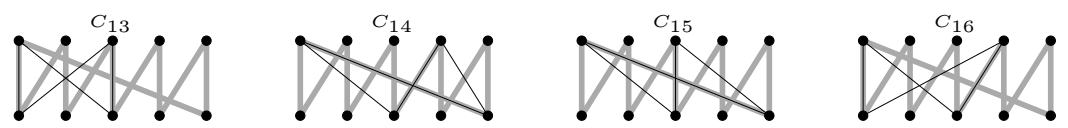

(c)

Figure 4: The sixteen basic circuits of $B$ (black edges) and the circuit $C$ (grey edges). For the sake of clearness we dropped the edges which are neither in the basic circuit nor in $C$.

Quasi-robust. The described basis had been shown to be quasi-robust in [12] in an elaborate manner.

Not cyclically robust. The circuit $C$ can be written as $C=\sum_{i=1}^{6} C_{i}$ and all the sums $C+C_{i}$ for $i=1, \ldots, 6$ are cycles with node degrees greater than 2 (marked by a circle), see Figure 4 (a). Hence, this cycle basis is not cyclically robust.

Not strictly quasi-robust. Looking at the remaining basic circuits, we observe that also $C_{7}$ to $C_{12}$ yield cycles with node degrees of 4 , Figure 4 (b), again marked by a circle. The intersection of $C_{13}$ to $C_{16}$ with $C$ is not a single path in each case, as can be seen in Figure 4 (c). In addition, $C+C_{13}$ and $C+C_{14}$ are disconnected. All in all, the cycle basis is not strictly quasi-robust.

Example 4.4 (Cycle basis, not even quasi-robust). The example of a cycle basis which is 
not even quasi-robust presented here had been inspired by a talk of Ostermeier [11]. ${ }^{1}$

The cycle basis is strictly fundamental and it is induced by the fat drawn tree in Figure 5 (a).

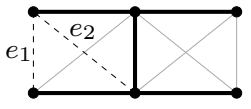

(a)

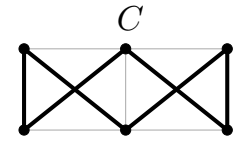

(b)
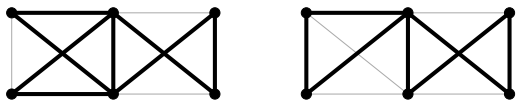

(c)

Figure 5: Graph with an inducing fundamental spanning tree (fat edges) and dashed chords $e_{1}$ and $e_{2}$ (a), a circuit $C$ (b), and sums of $C$ with two basic circuits generated by the chords $e_{1}$ and $e_{2}(\mathrm{c})$.

Not quasi-robust. Due to symmetry, we have to consider only the basic circuits induced by the dashed edges $e_{1}$ and $e_{2}$. In both cases, they add up with $C$ to a cycle that is not a circuit, see Figure 5 (c).

Example 4.5 (Cyclically robust cycle basis-not strictly quasi-robust). This example is a cycle basis on Wagner's graph $V_{8}$ which is cyclically robust, but not strictly quasi-robust. The strictly fundamental basis is indicated by the spanning tree which is highlighted in Figure 6 (a). The basic circuits are denoted at the chords. We use the notation from Example 4.2, i.e. $C_{i_{1}, \ldots, i_{k}}:=\sum_{j=1}^{k} C_{i_{j}}$.

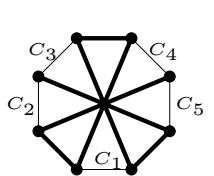

(a)

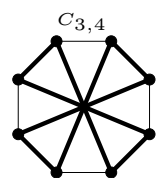

(b)

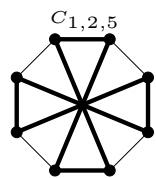

(d)

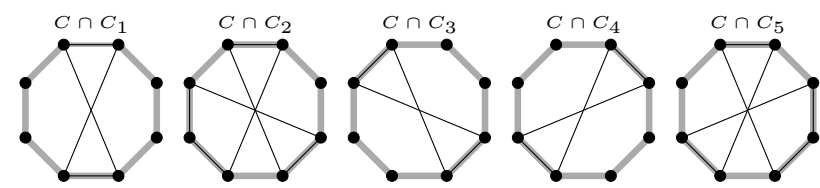

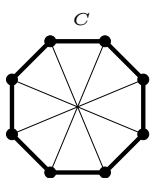

(c)

Figure 6: Wagner's graph $V_{8}$ with a fundamental spanning tree (a). The only two noncircuits in $V_{8}$ (b). The circuit $C$ (c). The intersections of $C$ (grey) with the five basic circuits do not form a single path (d), edges which are not in a basic circuit or in $C$ are dropped.

Cyclically robust. Wagner's graph $V_{8}$ is cubic which implies that every cycle is 2regular. The only critical cycles in $V_{8}$ are thus the two non-circuit pictured in Figure 6 (b). We provide the well-arranged sequences $S_{C_{3,4}+C_{1}}=S_{C_{1,3,4}}=\left(C_{1}, C_{3}, C_{4}\right), S_{C_{3,4}+C_{2}}=$ $S_{C_{2,3,4}}=\left(C_{2}, C_{3}, C_{4}\right)$, and $S_{C_{3,4}+C_{5}}=S_{C_{3,4}, 5}=\left(C_{4}, C_{5}, C_{3}\right)$ for the circuits which arise by adding a remaining basic circuit to $C_{3,4}$. For the cycle $C_{1,2,5}$ we give the sequences

\footnotetext{
${ }^{1}$ A similar example already appeared in [14].
} 
$S_{C_{1,2,5}+C_{3}}=S_{C_{1,2,3,5}}=\left(C_{1}, C_{2}, C_{3}, C_{5}\right)$ and $S_{C_{1,2,5}+C_{4}}=S_{C_{1,2,4,5}}=\left(C_{1}, C_{2}, C_{4}, C_{5}\right)$. In each of these sequences, every basic circuit appears at most once. This shows that the basis $B$ is cyclically robust.

Not strictly quasi-robust. To see that the basis is not strictly quasi-robust, consider the circuit $C$ in Figure 6 (c). Its intersection with each basic circuit does not form a single path. This is illustrated in Figure 6 (d).

Example 4.6 (Strictly quasi-robust cycle basis-not cyclically robust). The last example provides a graph with a cycle basis $B=\left\{C_{1}, \ldots, C_{6}\right\}$ which is strictly quasi-robust but not cyclically robust. As in Example 4.2 denote $C_{i_{1}, \ldots, i_{k}}:=\sum_{j=1}^{k} C_{i_{j}}$ for $2 \leq k \leq \nu$.

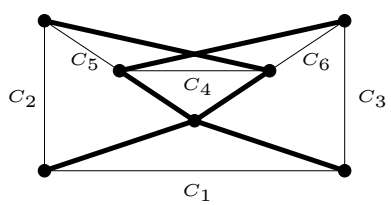

Figure 7: Graph with a fundamental spanning tree which induces a cycle basis that is strictly quasi-robust but not cyclically robust.

Strictly quasi-robust. Since $\nu=6$ we have to investigate $2^{6}-6-1=57$ cycles; the six basic circuits and the zero vector are not interesting. The 22 cycles listed below are not circuits.

$$
\begin{array}{llllllll}
C_{1,4}, & C_{2,3}, & C_{1,5,6}, & C_{4,5,6}, & C_{1,4,5,6}, & C_{2,3,5,6}, & C_{1,2,3,4,5}, & C_{1,2,4,5,6}, \\
C_{1,5}, & C_{1,4,5}, & C_{2,4,6}, & C_{1,2,4,6}, & C_{2,3,4,5}, & C_{2,4,5,6}, & C_{1,2,3,4,6}, & C_{1,3,4,5,6}, \\
C_{1,6}, & C_{1,4,6}, & C_{3,4,5}, & C_{1,3,4,5}, & C_{2,3,4,6}, & C_{3,4,5,6} & &
\end{array}
$$

For the remaining eleven circuits $C_{i, j}$ with $\left|\operatorname{supp}\left(C_{i, j}\right)\right|=2$ we may ignore the order of the basic circuits. The intersection of the two basic circuits is a path in each case, and thus, the sequences are strictly well-arranged. For the 24 circuits with at least three elements in their supports, we provide the following strictly well-arranged sequences.

$$
\begin{array}{llll}
\left(C_{1}, C_{2}, C_{3}\right), & \left(C_{1}, C_{3}, C_{6}\right), & \left(C_{4}, C_{6}, C_{3}\right), & \left(C_{1}, C_{2}, C_{5}, C_{6}\right), \\
\left(C_{1}, C_{2}, C_{4}\right), & \left(C_{3}, C_{4}, C_{2}\right), & \left(C_{5}, C_{6}, C_{3}\right), & \left(C_{4}, C_{6}, C_{3}, C_{1}\right), \\
\left(C_{1}, C_{2}, C_{5}\right), & \left(C_{3}, C_{5}, C_{2}\right), & \left(C_{1}, C_{2}, C_{3}, C_{4}\right), & \left(C_{5}, C_{6}, C_{3}, C_{1}\right), \\
\left(C_{1}, C_{2}, C_{6}\right), & \left(C_{3}, C_{6}, C_{2}\right), & \left(C_{1}, C_{2}, C_{3}, C_{5}\right), & \left(C_{1}, C_{2}, C_{3}, C_{5}, C_{6}\right), \\
\left(C_{1}, C_{3}, C_{4}\right), & \left(C_{4}, C_{5}, C_{2}\right), & \left(C_{1}, C_{2}, C_{3}, C_{6}\right), & \left(C_{1}, C_{2}, C_{3}, C_{6}, C_{5}, C_{4}, C_{1}\right), \\
\left(C_{1}, C_{3}, C_{5}\right), & \left(C_{2}, C_{5}, C_{6}\right), & \left(C_{4}, C_{5}, C_{2}, C_{1}\right), & \left(C_{1}, C_{2}, C_{3}, C_{5}, C_{6}, C_{4}\right) .
\end{array}
$$

Not cyclically robust. Figure 8 illustrates that the treated cycle basis is not cyclically robust. More precisely, look at the circuit $C_{2,3,4,5,6}$. For $i=2, \ldots, 6$, the cycles $C_{2,3,4,5,6}+C_{i}$ have nodes with degree greater than 2, marked by circles in Figure 8. Hence, this circuit does not admit a well-arranged sequence in which the circuits are pairwise disjoint.

\section{Relationship with fundamental bases}

One approach for a better understanding of strictly robust and cyclically robust cycle bases had been presented in [8]. Therein, the authors investigated the relationship between strictly 

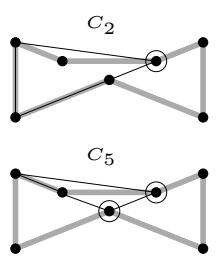
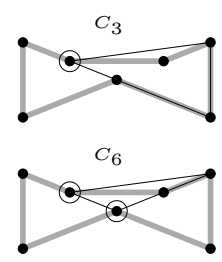

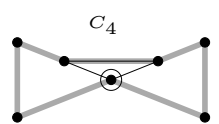

Figure 8: The circuit $C_{2,3,4,5,6}$ (grey) and the five basic circuits of its support (black edges).

robust, cyclically robust, and non-robust cycle bases on one hand, and strictly fundamental, weakly fundamental, and non-fundamental cycle bases on the other hand. Their motivation was the detailed exploration of strictly and weakly fundamental cycle bases which had been done in the years before. They concluded that robustness and fundamentality of cycle bases "are essentially unrelated concepts".

In more detail, they considered the combination (robustness, fundamentality), where robustness $\in\{$ "strictly robust", "robust", "non-robust" $\}$ and fundamentali$t_{y} \in\{$ "strictly fundamental", "weakly fundamental", "non-fundamental" $\}$. This immediately led to nine possibilities, and an example of a graph with an according cycle basis was presented in seven of these cases.

In this section, we follow up this line of research and provide for eight cases a graph with an appropriate cycle basis which is additionally minimum. For the ninth case, we are able to retire to a strictly quasi-robust cycle basis instead of a strictly robust one. However, this basis is not the minimum basis of the presented graph.

At the end of this section, we summarize our results in Table 2.

We start with three examples of strictly fundamental bases, that is the first column in Table 2. Two of them are taken from [8], the third one correlates to the basis in Example 4.5. Due to Lemma 2.1, all bases can be made minimum.

Example 5.1 (Strictly fundamental-strictly robust). This example is directly taken from [8]. To be more accurate, we deal with the complete graph $K_{n}$ and the cycle basis $B_{n}$ which is induced by the complete bipartite graph $K_{1, n-1}$ as fundamental spanning tree. It is strictly robust as shown in [6]. With a weighting assigned according to Lemma 2.1 it is also the unique minimum cycle basis.

We decided to present this example here because it constitutes a whole class of graphs and cycle bases with the required properties. On the other hand, also the triangle graph in Example 4.1 could have served as an example at this place.

Example 5.2 (Strictly fundamental—not strictly robust—cyclically robust). Wagner's graph $V_{8}$ and the cycle basis which had already been presented in Example 4.5 provide the necessary properties for this example. We remark that this example eliminates one of the two question marks in [8] where the authors conjectured the existence of such an example.

Example 5.3 (Strictly fundamental—not cyclically robust). Again, we borrow the example given in [8] which is called there "Ostrowski's basis". It is simply the $K_{5}$ with a path consisting of four edges as fundamental spanning tree. This spanning tree induces a basis consisting of three triangles, two quadrangles, and one pentagon. To verify that the basis is non-robust, take a look at the circuit $C$ which is the sum of the three triangles and the two quadrangles. The sum of $C$ with each of these basic circuits constitutes a non-circuit. 
Similarly to Example 5.2, we could have borrowed the graph with a non-robust cycle basis from Example 4.4. Anyway, we used Ostrowski's basis at this place because there is an easy way to construct an infinite class of graphs and cycle bases with the required properties. More precisely, we speak about the family of complete graphs with an odd number of vertices. For such a graph $G_{k}=\left(V_{k}, E_{k}\right)$ with $V_{k}=\left\{v_{0}, v_{1}, \ldots, v_{2 k}\right\}$ we choose the path $\left(v_{0}, v_{1}, \ldots, v_{2 k}\right)$ as inducing spanning tree for the strictly fundamental cycle basis. As a certificate for the non-robustness, we provide the circuit $C_{k}=$ $\bigcup_{i=0}^{2 k}\left\{v_{i} v_{i+2}\right\}=\sum_{i=0}^{2 k}\left\{v_{i} v_{i+1}, v_{i+1} v_{i+2}, v_{i+2} v_{i}\right\}$, where the indices are taken modulo $2 k+1$. Adding one basic circuit $C_{k}^{i}=\left\{v_{i} v_{i+1}, v_{i+1} v_{i+2}, v_{i+2} v_{i}\right\}$ to $C_{k}$ results in a cycle $C_{k}^{\prime}$ with $\operatorname{deg}_{C_{k}^{\prime}}\left(v_{i+1}\right)=4$. In Figure 9 , the graph $G_{3}$ is given as an example.

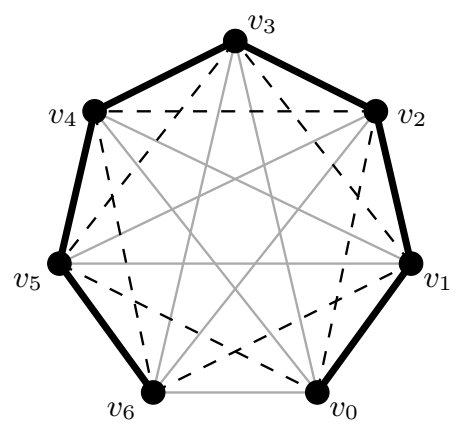

Figure 9: The graph $G_{3}$, the inducing spanning tree (fat edges), and the circuit $C_{k}$ (dashed edges).

We continue with three examples which are weakly fundamental but not strictly fundamental. In Table 2, these examples appear in the second column. One example is taken from [9]. For the other two, we destroy the strictly fundamentality of the according examples above by gluing suitable graphs together. In doing so, we keep in mind that we want the bases to stay minimum.

Example 5.4 (Not strictly fundamental-weakly fundamental-strictly robust). The additional demand for a minimum cycle basis prevents us from simply copying the according example in [8]. Instead, we copy Example 11.2 from [9], which deals with the sunflower graph SF (3), depicted in Figure 10. Therein, it served as an example for a 2-basis which is not strictly fundamental, where for a 2-basis, each edge is contained in at most two basic cycles.

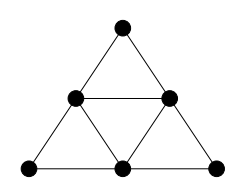

Figure 10: The sunflower graph $\mathrm{SF}(3)$.

The cycle basis $B$ consisting of the only four triangles is the unique minimum cycle basis. Each edge of the middle triangle is contained in another circuit of $B$, thus, $B$ is not strictly 
fundamental. But since the basis is a 2-basis, it is weakly fundamental, see e.g. [9], and strictly robust due to [2].

Example 5.5 (Not strictly fundamental-weakly fundamental-not strictly robust-cyclically robust). The idea in this example is to adapt Wagner's graph and its cycle basis presented in Examples 4.5 resp. 5.2 such that it is not strictly fundamental anymore. To do this, we append a further path $\left(v_{1}, v_{2}, v_{3}\right)$ at the two adjacent vertices $v_{1}$ and $v_{3}$ at the right hand side of the graph, see Figure 11.

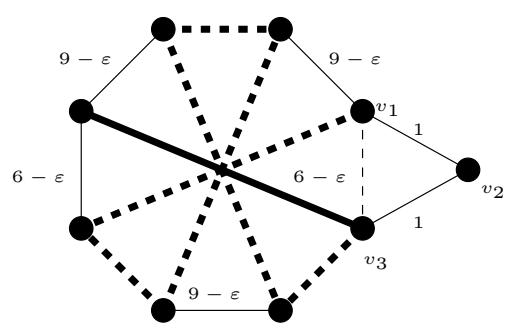

Figure 11: The modified Wagner's graph with a partial spanning tree (fat edges) and a circuit without private edge (dashed).

The weights of the graph are assigned according to Lemma 2.2. The second statement of this lemma does hold for $v_{1} v_{3}$, i.e. $w\left(v_{1} v_{3}\right)<\operatorname{dist}_{T}\left(v_{1}, v_{3}\right)$. For the new edges set $w\left(v_{1} v_{2}\right)=w\left(v_{2} v_{3}\right)=1$. To yield the cycle basis, inherit the basic circuits from the original example and append the circuit $C_{6}=\left(v_{1} v_{2}, v_{2} v_{3}, v_{3} v_{1}\right)$. Remark that the weights of the old edges were chosen according to Lemma 2.2 and that $C_{6}$ is the shortest circuit which contains the new vertex $v_{2}$. Hence, the obtained cycle basis is minimum.

The basis is not strictly robust for the same reasons as in Example 4.5. On the other hand, assume that a circuit $C$ in this graph contains the vertex $v_{2}$. A well-arranged sequence for $C$ can be achieved by concatenating $C_{6}$ with the well-arranged sequence of $C+C_{6}$, hence, the basis is cyclically robust. Finally, the cycle basis is not strictly fundamental, since the dashed basic circuit does not have a private edge. But it is weakly fundamental because Inequality (2.1) holds for each permutation $\pi$ with $C_{\pi(6)}=C_{6}$.

Example 5.6 (Not strictly fundamental-weakly fundamental—not cyclically robust). Similarly to the example above, we destroy the strictly fundamentality of Ostrowski's basis of the $K_{5}$. We also could have used Lemma 2.2 and could have constructed a graph by simply appending a path of length 2 as in Example 5.5. Anyway, we decided to provide a larger example in favor of an integer weight function.

Remember that the basis of this graph was induced by a path of four edges as fundamental spanning tree. There is one edge between the end nodes of the path, denote it $e_{P}$. Now take three copies of $K_{5}$ and assemble them in a way such that the three copies of $e_{P}$ constitute a triangle, add a vertex and connect it to the three corners of the triangle. See Figure 12 for the construction. The edge weights in the three copies of $K_{5}$ are assigned according to Lemma 2.1, the three new edges get the weight 2. Again, the fat edges get weight 1.

To get a cycle basis for the merged graph, combine the cycle bases of the three copies and append the three new triangles with weight 8 , i.e. the triangles constituted by two new edges and one copy of $e_{P}$. The $\nu=21$ shortest circuits have weight 8 , hence, the combined 


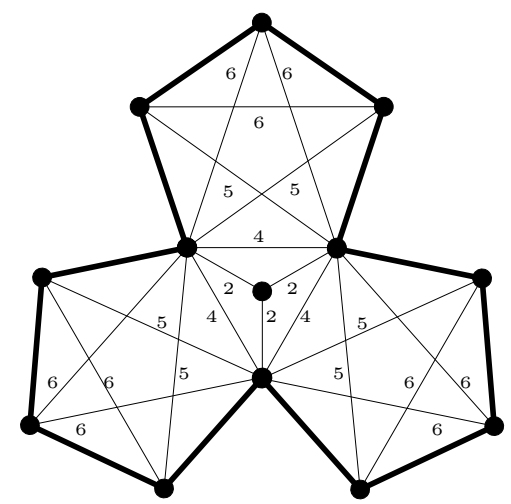

Figure 12: Three merged copies of $K_{5}$ with Ostrowski's bases.

cycle basis is minimum. It is not robust because Ostrowski's basis is not. It is not strictly fundamental since the circuits induced by $e_{P}$ in each $K_{5}$ do not have private edges, as well as the three new triangles. In the end, it is weakly fundamental. Permute the basis such that the three new triangles appear at first, followed by the three circuits induced by the copies of $e_{P}$.

The last three examples present non-fundamental cycle bases, listed in the third column of Table 2. Two of them are again borrowed from [9].

Example 5.7 (Not weakly fundamental-strictly quasi-robust). Unfortunately, we were not able to give an example of a minimum non-fundamental cycle basis which is strictly robust. But we provide a strictly quasi-robust one, at least. Therefore, look at the graph depicted in Figure 13 and the indicated cycle basis.

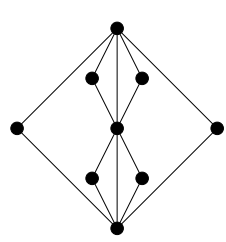

(a)
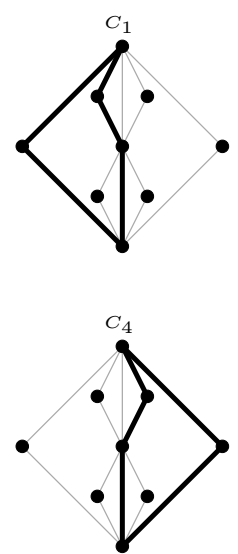
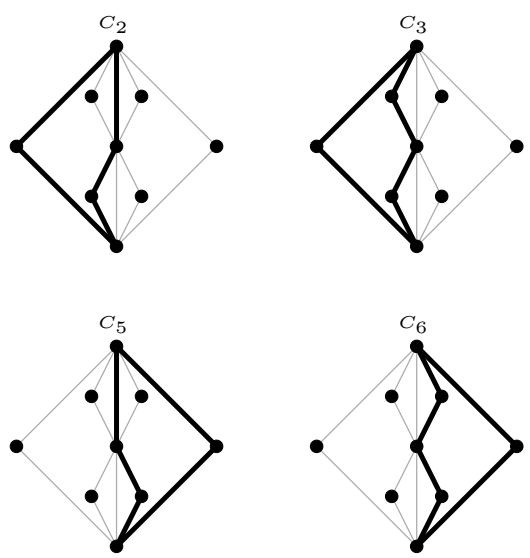

(b)

Figure 13: A graph (a) and a non-fundamental cycle basis which is strictly quasi-robust, but not strictly robust (b).

The basis is non-fundamental since each edge is contained in at least two basic circuits. 
To see that it is strictly quasi-robust, we take a look at $2^{6}-6-1=57$ cycles, analogous to Example 4.6. Among these cycles, there are 38 which do not constitute circuits. For the other 19 circuits, we provide the strictly well-arranged sequences below.
$\left(C_{1}, C_{3}\right)$
$\left(C_{2}, C_{3}, C_{5}\right)$
$\left(C_{1}, C_{3}, C_{4}, C_{6}, C_{2}\right)$,
$\left(C_{2}, C_{3}\right)$,
$\left(C_{6}, C_{5}, C_{2}\right)$,
$\left(C_{3}, C_{1}, C_{4}, C_{6}, C_{5}, C_{2}, C_{3}\right)$,
$\left(C_{4}, C_{6}\right)$,
$\left(C_{6}, C_{5}, C_{4}\right)$,
$\left(C_{6}, C_{5}, C_{2}, C_{3}, C_{1}\right)$,
$\left(C_{5}, C_{6}\right)$,
$\left(C_{1}, C_{3}, C_{4}, C_{6}\right)$,
$\left(C_{3}, C_{2}, C_{1}\right)$,
$\left(C_{2}, C_{3}, C_{5}, C_{6}\right)$,
$\left(C_{1}, C_{3}, C_{4}, C_{6}, C_{5}\right)$,
$\left(C_{1}, C_{3}, C_{4}\right)$,
$\left(C_{6}, C_{4}, C_{1}, C_{3}, C_{2}, C_{5}, C_{6}\right)$,
$\left(C_{2}, C_{3}, C_{5}, C_{6}, C_{4}\right)$,
$\left(C_{6}, C_{4}, C_{1}\right)$,
$\left(C_{6}, C_{4}, C_{1}, C_{3}, C_{2}, C_{5}\right)$

For the circuits which belong to the bold written sequences, there are no strictly wellarranged sequences in which all circuits are pairwise disjoint. Thus, the cycle basis is strictly quasi-robust, but not strictly robust.

Example 5.8 (Not weakly fundamental-not strictly robust_cyclically robust). The cycle basis in this example is borrowed from [9] where it serves as an example of a minimum cycle basis which is not integral ${ }^{2}$. It is a basis of the generalized Petersen graph $P_{11,4}$, see Figure 14.

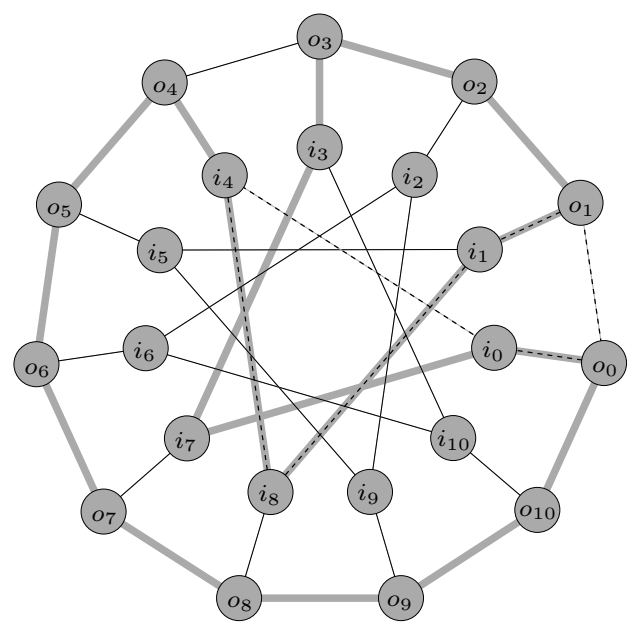

Figure 14: Generalized Petersen graph $P_{11,4}$ with the basic circuit $C_{1}$ (dashed) and the circuit $C_{1,4,12}=C_{1}+C_{4}+C_{12}$ (grey).

The discussed basis $B$ contains the circuits $C_{j+1}=\left(o_{j} i_{j}, i_{j} i_{j+4}, i_{j+4} i_{j+8}, i_{j+8} i_{j+1}\right.$, $\left.i_{j+1} o_{j+1}, o_{j+1} o_{j}\right)$ for $j=0, \ldots, 10$ where the indices are taken modulo 11, and the circuit $C_{12}=\left\{o_{0} o_{1}, \ldots, o_{9} o_{10}, o_{10} o_{0}\right\}$. In the figure above we emphasized the circuit $C_{1}$ with dashed edges. With the weights $w\left(o_{j} o_{j+1}\right)=4, w\left(i_{j} i_{j+4}\right)=5$, and $w\left(o_{j} i_{j}\right)=12$, again for $j=0, \ldots, 10$ and again modulo 11 , this basis becomes the unique minimum one, see [9].

\footnotetext{
${ }^{2}$ For the definition of integral cycle bases we refer to [9].
} 
Each edge $i_{j} i_{j+4}$ is contained in three basic circuits, all other edges in exactly two basic circuits. This shows the non-fundamentality of the basis. To see that it is not strictly robust, consider for example the circuit $C_{1,4,12}=C_{1}+C_{4}+C_{12}$ whose cuts with $C_{1}, C_{4}$, and $C_{12}$ do not form a single path in each case.

It remains to show that the basis is cyclically robust. This was done by a small program implemented in C++ using LEDA ([10]). The program tested for each of the $2^{12}$ linear combinations if it constitutes a circuit $C$, and if so, if there is a circuit $C_{j} \in \operatorname{supp}(C)$ such that $C+C_{j}$ is a circuit. This applied to each circuit and thus, the cycle basis is cyclically robust.

Example 5.9 (Not weakly fundamental —not cyclically robust). To construct cycle bases of a biconnected graph which are neither robust nor fundamental, the authors in [8] suggest the following operation. Given a 2-connected graph $G^{\prime}$ with a non-robust cycle basis $B^{\prime}$ and a 2-connected graph $G^{\prime \prime}$ with a non-fundamental cycle basis $B^{\prime \prime}$, construct a graph $G$ by identifying two arbitrary edges of $G^{\prime}$ and $G^{\prime \prime}$. The basis $B=B^{\prime} \cup B^{\prime \prime}$ is a basis of $G$. However, even if $B^{\prime}$ and $B^{\prime \prime}$ are the minimum cycle bases of $G^{\prime}$ and $G^{\prime \prime}$, respectively, it is not guaranteed that $B$ is a minimum cycle basis of $G$. In contrast to this construction, we propose Champetier's graph with its minimum cycle basis as a representative for a minimum non-robust and non-fundamental cycle basis.

Also this graph and the cycle basis are taken from [9]. In his Example 11.7, Liebchen considered Champetier's graph whose unique minimum cycle basis is integral but neither weakly fundamental nor totally unimodular. In Champetier's original paper [1], it served as a counter-example of a conjecture expressed in [3]: "If $G$ is null-homotopic (i.e., if every cycle of $G$ is the modulo 2 edge sum of triangles), there is an edge $e$ of $G$ such that $G \backslash e$ is still null-homotopic." Champetier's graph is visualized in Figure 15.

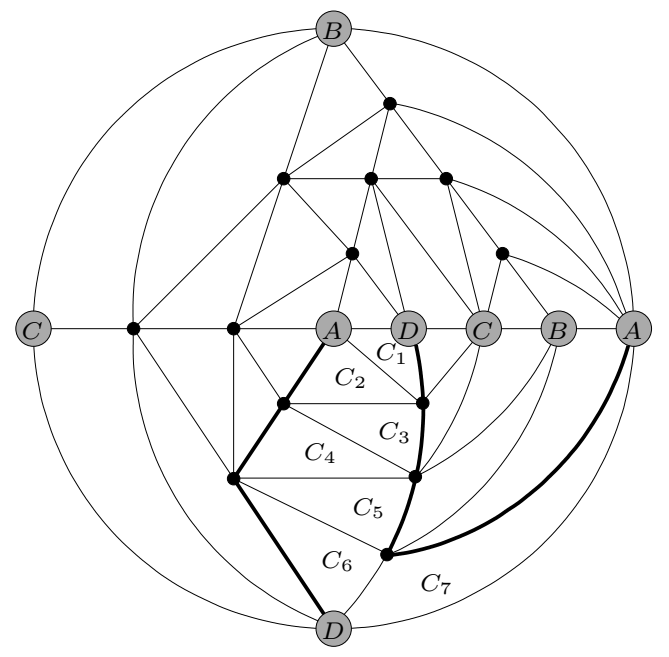

Figure 15: Champetier's graph and a certificate for the non-robustness of the minimum cycle basis.

Champetier's graph arises from the embedding by identifying the vertices $A, B, C$ and $D$ with their copies. The cycle basis we deal with is formed by the 36 triangles in the 
embedded version. This basis is minimum since there is neither a further triangle which is not the boundary of a face in the embedding in Figure 15 nor a path of length 3 between two copies of one of the vertices $A$ to $D$. After the vertex identifications, such a path would also compose to a triangle. Hence, the basic circuits are the only triangles.

Since each edge is contained in two triangles at least, the basis is non-fundamental. As a proof for the non-robustness, we take the same certificate as in Example 11.7 in [9], i.e. the circuit $C=\sum_{i=1}^{7} C_{i}$, indicated in Figure 15 by two paths. In fact, $C+C_{i}$ does not form a circuit for $i=1, \ldots, 7$. This shows that the basis is non-robust.

Table 2 summarizes the results of this section. It has been inspired by the Venn diagram in [8] which also illustrates the relationship between fundamental and robust cycle bases. In the table, we contrast our results with the results listed there. New examples and improvements are emphasized in italic.

Table 2: Overview of the results in this section.

\begin{tabular}{|c|c|c|c|c|}
\hline & strictly fundamental & weakly fundamental & non-fundamental & \\
\hline \multirow{2}{*}{ 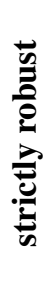 } & $\begin{array}{l}K_{n} \text { with } K_{1, n-1} \\
\text { as fund. sp. tree } \\
\text { minimum basis }\end{array}$ & $\begin{array}{l}\text { Fig. } 2 \text { in [8] } \\
\text { basis not minimum }\end{array}$ & $?$ & [8] \\
\hline & as above & $\begin{array}{l}\text { sunflower graph } S F(3) \\
\text { minimum basis }\end{array}$ & $\begin{array}{l}\text { Ex. } 5.7 \text {, basis only } \\
\text { strictly quasi-robust } \\
\text { basis not minimum }\end{array}$ & $\begin{array}{l}\text { this } \\
\text { paper }\end{array}$ \\
\hline \multirow{2}{*}{ 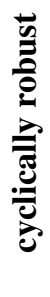 } & $?$ & $\begin{array}{l}\text { Kainen's basis of } K_{4} \\
\text { basis not minimum }\end{array}$ & $\begin{array}{l}\text { non-fundamental } \\
\text { basis of the } 4-\text { wheel } \\
\text { basis not minimum }\end{array}$ & [8] \\
\hline & $\begin{array}{l}\text { Wagner's graph with } \\
\text { a } P_{7} \text { as fund. sp. tree } \\
\text { minimum basis }\end{array}$ & $\begin{array}{l}\text { Wagner's graph joined } \\
\text { up with a triangle } \\
\text { minimum basis }\end{array}$ & $\begin{array}{l}\text { Petersen graph } P_{11,4} \\
\text { minimum basis }\end{array}$ & $\begin{array}{l}\text { this } \\
\text { paper }\end{array}$ \\
\hline \multirow{2}{*}{ 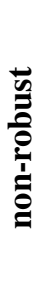 } & $\begin{array}{l}K_{5} \text { with } P_{4} \\
\text { as fund. sp. tree } \\
\text { basis not minimum }\end{array}$ & $\begin{array}{l}\text { Vogt's example } \\
\text { basis not minimum }\end{array}$ & $\begin{array}{l}\text { merging non-rob. basis } \\
\text { with non-fund. basis } \\
\text { basis not minimum }\end{array}$ & [8] \\
\hline & $\begin{array}{l}\text { as above } \\
\text { basis minimum with } \\
\text { a suitable weighting }\end{array}$ & $\begin{array}{l}\text { three merged } K_{5} \\
\text { minimum basis }\end{array}$ & \begin{tabular}{l} 
Champetier's graph \\
\hdashline- mimum basis $^{-}$
\end{tabular} & $\begin{array}{l}\text { this } \\
\text { paper }\end{array}$ \\
\hline
\end{tabular}

\section{Concluding remarks}

In this paper, we considered robust cycle bases and isolated strictly and cyclically robust cycle bases, as well as the newer concepts of quasi-robust and strictly quasi-robust cycle bases from each other. We did this by giving suitable examples. Since each of our cycle bases is the uniquely minimum one of its graph, and hence each type of robust cycle basis 
comes along with its own minimization problem, we can view the classification of robust cycle bases as completed.

A second focus was the continuation of the comparison between robust and fundamental types of cycle bases. We were able to further fill the Venn diagram of robust and fundamental cycle bases given in [8], where we demanded in addition that the provided cycle basis is minimum. Our results were summarized in a table which has only one missing item. We could not present a minimum cycle basis which is non-fundamental and strictly robust, but could provide an example of a cycle basis which is strictly quasi-robust, at least.

Despite all, there is still plenty of work to do in the field of robust cycle bases. For example, it is still unknown whether each graph provides a strictly robust cycle basis, or a cycle basis of any other robust type, at least. Furthermore, there is nothing known about the complexity of recognition and construction of robust cycle bases.

\section{References}

[1] C. Champetier, On the null-homotopy of graphs, Discrete Math. 64 (1987), 97-98, doi:10. 1016/0012-365x(87)90244-5.

[2] U. Doḡrusöz and M. S. Krishnamoorthy, Enumerating all cycles of a planar graph, Parallel Algorithms Appl. 10 (1996), 21-36, doi:10.1080/10637199608915603.

[3] P. Duchet, M. Las Vergnas and H. Meyniel, Connected cutsets of a graph and triangle bases of the cycle space, Discrete Math. 62 (1986), 145-154, doi:10.1016/0012-365x(86)90115-9.

[4] P. M. Gleiss, Short Cycles, Ph.D. thesis, Universität Wien, 2001, https://www.tbi. univie.ac.at/papersold/papers/Abstracts/pmg_diss.pdf.

[5] D. Hartvigsen and E. Zemel, Is every cycle basis fundamental?, J. Graph Theory 13 (1989), 117-137, doi:10.1002/jgt.3190130115.

[6] P. C. Kainen, On robust cycle bases, Electron. Notes Discrete Math. 11 (2002), 430-437, doi: 10.1016/s1571-0653(04)00087-3.

[7] P. C. Kainen, Cycle construction and geodesic cycles with application to the hypercube, Ars Math. Contemp. 9 (2015), 27-43, http: / / amc-journal.eu/index.php/amc/ article/view/450.

[8] K. Klemm and P. F. Stadler, A note on fundamental, non-fundamental, and robust cycle bases, Discrete Appl. Math. 157 (2009), 2432-2438, doi:10.1016/j.dam.2008.06.047.

[9] C. Liebchen, Periodic Timetable Optimization in Public Transport, Ph.D. thesis, Technische Universität Berlin, 2006.

[10] K. Mehlhorn and S. Näher, LEDA: A Platform for Combinatorial and Geometric Computing, Cambridge University Press, Cambridge, 1999, https://people.mpi-inf.mpg.de/ $\sim$ mehlhorn/LEDAbook.html.

[11] P.-J. Ostermeier, Quasi-robust Cycle Spaces, 2009, http://www.bioinf. uni-leipzig.de/conference-registration/0 9herbst/talks/401_ Ostermeier.pdf (accessed on 4 October 2017).

[12] P.-J. Ostermeier, M. Hellmuth, K. Klemm, J. Leydold and P. F. Stadler, A note on quasi-robust cycle bases, Ars Math. Contemp. 2 (2009), 231-240, http: / / amc- journal . eu/ index. $\mathrm{php/amc/article/view/104.}$

[13] A. Reich, Cycle Bases of Graphs and Spanning Trees with Many Leaves, Ph.D. thesis, Brandenburgische Technische Universität Cottbus - Senftenberg, 2014, https : / / opus 4 . kobv . de/opus4-btu/files/2966/Dissertation_Reich.pdf. 
[14] M. M. Sysło, On cycle bases of a graph, Networks 9 (1979), 123-132, doi:10.1002/net. 3230090203.

[15] G. Wünsch, Coordination of Traffic Signals in Networks and Related Graph Theoretical Problems on Spanning Trees, Ph.D. thesis, Technische Universität Berlin, 2008. 